\title{
Classification of Alcoholic and Non-Alcoholic EEG Signals Based on Sliding-SSA and Independent Component Analysis
}

\author{
Shrey Agarwal, Muhammad Zubair, Student Member, IEEE
}

\begin{abstract}
Alcoholism is a widely affected disorder that leads to critical brain deficiencies such as emotional and behavioural impairments. One of the prominent sources to detect alcoholism is by analysing Electroencephalogram (EEG) signals. Previously, most of the works have focused on detecting alcoholism using various machine and deep learning algorithms. This paper has used a novel algorithm named Sliding Singular Spectrum Analysis (S-SSA) to decompose and de-noise the EEG signals. We have considered independent component analysis (ICA) to select the prominent alcoholic and non-alcoholic components from the preprocessed EEG data. Later, these components were used to train and test various machine learning models like SVM, KNN, ANN, GBoost, AdaBoost and XGBoost to classify alcoholic and nonalcoholic EEG signals. The sliding SSA-ICA algorithm helps in reducing the computational time and complexity of the machine learning models. To validate the performance of the ICA algorithm, we have compared the computational time and accuracy of ICA with its counterpart, like principal component analysis (PCA). The proposed algorithm is tested on a publicly available UCI alcoholic EEG dataset. To verify the performance of machine learning models, we have calculated various metrics like accuracy, precision, recall and F1 score. Our work reported the highest accuracy of $98.97 \%$ with the XGBoost classifier. The validation of the proposed method is done by comparing the classification metrics with the latest state-of-the-art works.
\end{abstract}

Index Terms_-Alcoholism, EEG Signals, Sliding Singular Spectrum Analysis, Independent Component Analysis, Machine Learning Models

\section{INTRODUCTION}

Electroencephalogram signals are the most important factors to measure the electrical activity of brain, which helps in detecting various diseases like epileptic seizures, sleep disorders, alcoholism, Alzheimer's disease, and Parkinson's disease [1]. Nowadays, the most concerning disease in the society is alcoholism. This disease is the main cause for various incidents like road accidents, harassments, rapes, and violence. As per the reports by world health organisation (WHO), humanity witnesses over 3.3 million deaths a year because of excessive alcoholism [2]. It is seen that alcohol addicts have problem with decision making, memory loss and behavioural changes.

The detection of liquor in subjects is a difficult task on the grounds, because the standard gadgets depend on the smell of the beverage, which isn't generally precise. As of late, it has been exhibited that EEG signals can be utilized as a symptomatic tool in the assessment of subjects with liquor addiction.

In past works, classification of alcoholic and non-alcoholic EEG signals was performed using gamma signals [3]. These

This paragraph of the first footnote will contain the date on which you submitted your paper for review.

Shrey Agarwal is with the Department of Computer Science Engineering, Kalinga Institute of Industrial Technology, Bhubaneshwar, Orissa, India (e-mail: shreyagarwal1111@gmail.com).

Muhammad Zubair is with the Department of Electronics and Communication Engineering, Prasad V. Potluri Siddhartha Institute of Technology, Vijayawada, AP, India (e-mail:muhammadzub17@gmail.com). signs were separated from the EEG signal, and a few boundaries were determined as the quality of the EEG signal. Another investigation in [4] utilized Wavelet Packet Decomposition (WPD), Principal Component Analysis (PCA), and BackPropagation Neural-Network (BP-NN) enhanced by hereditary calculations for the grouping of alcoholic EEG. This work achieved an highest accuracy of 94\% on the third level of decomposition. In [5] Bajaj et al. reported Short Time Fourier Transform (STFT) and non-negative least squares classifier (NNLS) for the detection of alcoholic EEG signals. In [6] Kousarrizi et al. implemented Wavelet Transform (WT) based features extraction technique and classified the alcoholic EEG signals using SVM. Sun et al. [7] reduced the dimensions of original EEG data by employing Principal Component Analysis (PCA). They used WT based features for analysis of alcoholic and control EEG signals. In [8], alcoholic and normal EEG signals were classified using correlation dimension based features. In [9] Acharya et al. have proposed a method in which for the SVM classifier non-linear features were used as input for the classification of EEG signals as alcoholic or normal. Faust et al. [10] reported a method in which various machine learning algorithms were used with wavelet packet decomposition for the extraction of energy measures, and through this alcoholic EEG signals could be identified. For the classification of alcoholic and control EEG signals, the features used were the fast fourier transform (FFT) and auto regressive (AR) method-based power density [11].

As discussed in the above works, we can observe that all the 
works are more inclined towards various wavelet transform, PCA, STFT and other energy based features. In this work, we have introduced S-SSA to decompose and de-noise the EEG signals. For the purpose of feature extraction, we implemented ICA to extract independent components from the alcoholic and non-alcoholic EEG signals. These independent components are used as an input for the binary classification of both the signals using six different machine learning models (SVM, KNN, AdaBoost, GBoost, ANN and XGBoost).

\section{LITERATURE REVIEW}

Since past few years, researchers have explored various statistical and machine learning approaches to detect the problem of alcoholism. Jiajie et al. [21] designed a GUI for feature extraction and classification of two classes. They have utilised UCI database for the implementation of their work. This is attained by using electrodes frontal, frontal polar and central for collecting raw EEG data and then calculating approximate entropy, sample entropy, standard deviation and mean. The highest accuracy was reported as $95 \%$ by quadratic SVM for the detection of alcoholic EEG signals. The GUI system is used as a proper clinical resolution support structure by clinicians, which is economical and efficient. It also helps rehabilitation centres in being acquainted with the subject.

In [22] Izawa et al. have proposed a state-of-the-art and dependable alcoholic-intake identification system that examines photoplethysmogram (PPG) signal captured from the subject. The method used in this paper uses harmonic intensity ratio of the PPG signal and the oxygen-saturation level ( $\mathrm{SpO} 2)$ in the artery. Their method can create a particular SIRZ which can cancel the outcome of smoking and exercise, and this is useful as a large amount of the data of alcohol consumption lie within the specified SIRZ and thus offer a significant prospects in PPG systems that can trace the usage of alcohol.

Anuragi et al. [23] have used machine learning models based on flexible analytical wavelets transform (FAWT) for automated alcoholic signal detection. Using FAWT, EEG signals are decomposed into elaborate and estimated wavelet coefficients. The statistical features such as standard deviation, mean, skewness, kurtosis and shannon entropy are pulled out from the determined wavelet coefficients. The features are then fed as input to numerous machine learning models which are SVM, Least Square-Support Vector Machine (LS-SVM) and Naive Bayes classifier. The performance of models were trained and tested using 10 -fold cross validation by calculating all the required measures such as sensitivity, accuracy, Fmeasure, specificity, Matthew's correlation coefficient (MCC) precision, and ROC. The outcome suggests that LS-SVM using polynomial kernel had the best accuracy of $99.17 \%$, Specificity 99.44\% and Sensitivity as $99.17 \%$.

Moreover, Patidar et al. [24] have discussed a novel way for identification of alcoholism using Tunable-Q Wavelet Transform (TQWT), which is based on attributes coming from EEG signals. The attribute extraction is performed using extracted Centered Correntropy (CC) and TQWT based decomposition from the fourth decomposed detail sub-band. 10-fold crossvalidation LS-SVM is implemented on PCA which is used for feature depletion followed to classify EEG signals as alcoholic or normal. Sensitivity of $96.53 \%$, average accuracy of $97.02 \%$, Matthews correlation coefficient of 0.9494 for Q-factor (Q) varying between 3 and 8 using Radial Basis Function (RBF) kernel and specificity of $97.50 \%$ was found. Also using three clinically significant features, they have formed a new Alcoholism Risk Index (ARI) to differentiate the given classes by means of a single number.

Siuly et al. [25] introduced an optimum allocation based sampling (OAS) method to find the most likely typical data points from each single time-window of every EEG signal taking into account the minimum variability of the observations. Statistical features are found from every set of class by amalgamating all representative samples of every timewindow. The Mann-Whitney U test is used to examine whether each of the attribute is contributing independently for the binary classification. Four machine learning methods SVM, decision table, logistic regression and k-NN are considered for assessing the efficacy of the OAS-based features.

Similarly, Acharya et al. [26] presents an analysis of the familiar features of EEGs acquired from PWA and put forwards that the effect of alcoholism on the brain can be determined by computer-aided analysis of EEGs through extracting the diminutive differences in the EEG signals that can differentiate the EEGs of PWA from those of not affected people. This is attained through sensible amalgamation of signal processing methods such as non-linear dynamics, wavelet, pattern recognition and chaos theory and classification techniques. A CAD system provides the following unbiased results. Independent of the user, ease of use, efficiency, low cost and reproducible results.

All the alcoholism related works discussed above are based on FFT, AR models, STFT, PCA, WPD and MCC's. The features were extracted using these techniques and classification is performed by using SVM, LS-SVM and KNN. The major drawback of all these works is that they have not reported about the computational time for detection of alcoholism. As we know in medical field the most important factors are
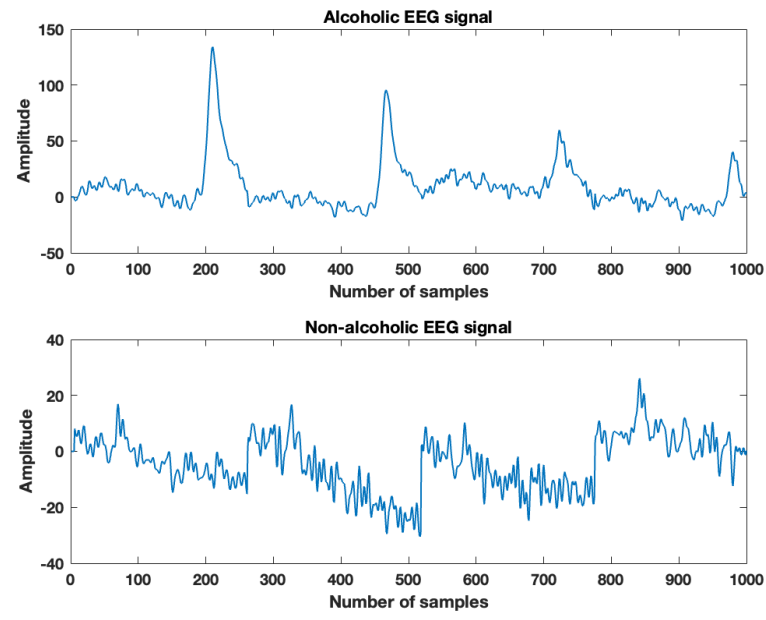

Fig. 1: Alcoholic and non-alcoholic EEG signals 


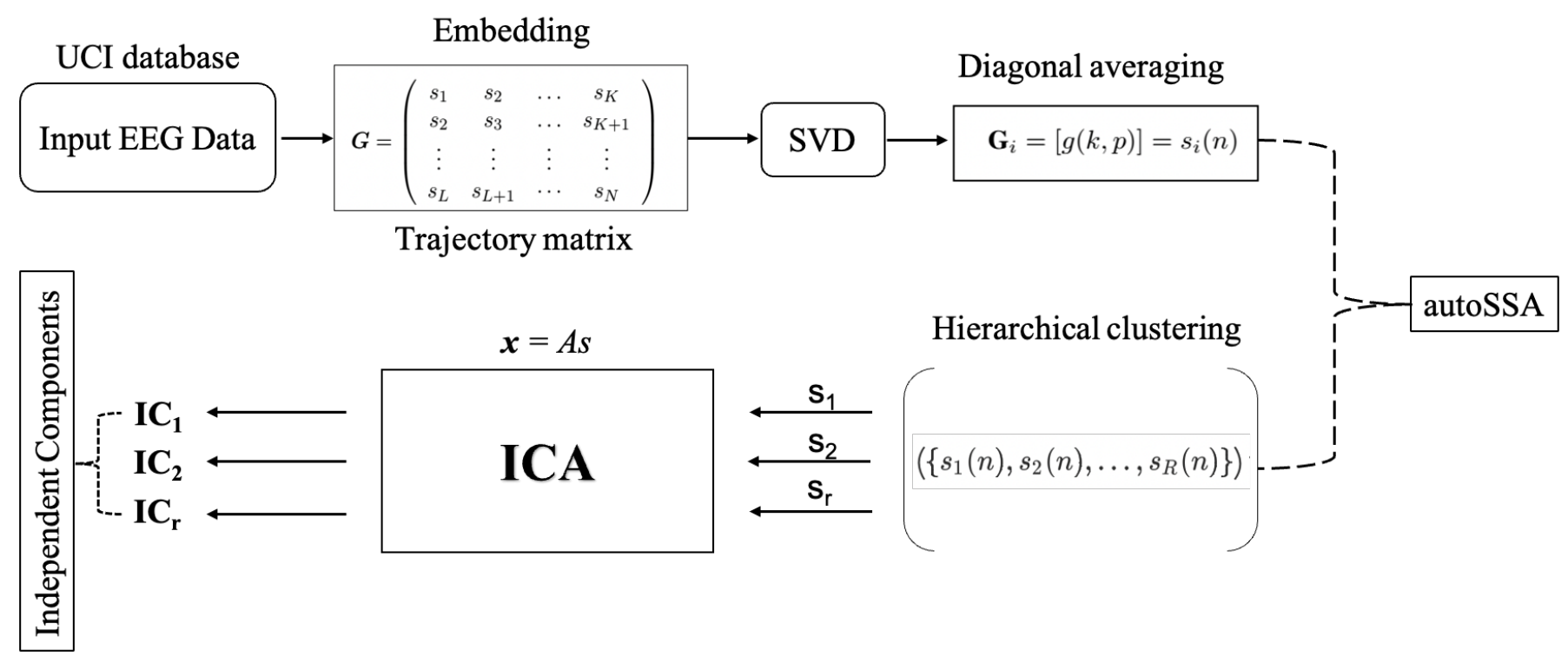

Fig. 2: Hierarchy of proposed sliding SSA-ICA algorithm

time and efficiency. Hence, more work is needed to classify alcoholism by considering the computational time and complexity of the machine learning models. To overcome the stated problem, we have proposed an algorithm based on sliding SSA-ICA which helps in identifying the alcoholic components from the EEG signals and reduces the computational time and complexity of the machine learning models.

\section{Proposed Methodology}

The proposed method mainly consist of four steps, (i) Preprocessing of EEG signals using sliding SSA, (ii) Calculating independent components of alcoholic and non-alcoholic EEG signals using ICA, (iii) Classification of alcoholic and nonalcoholic EEG signals, (iv) Calculation of computational time for various machine learning models. The brief hierarchy of first two steps is shown in Fig. 2.

\section{A. Data description}

The dataset for the current research is from UCI [12], the EEG dataset is from the Neurodynamic Laboratory at the State University of New York. It has 77 subjects which are diagnosed with alcoholism and 45 control subjects making a total of 122 subjects. 120 separate trials were performed for every subject. All labels were labelled alcoholism for the trials in which the subject was alcoholic. All trials had 64 electrodes placed on the subjects' scalps for 1 second and were sampled at $256 \mathrm{~Hz}$. The graphical representation of the alcoholic and non-alcoholic EEG signals is shown in Fig. 1.

\section{B. Sliding singular spectrum analysis (S-SSA)}

The S-SSA can be derived from the standard SSA algorithm. The SSA algorithm is a mixture of two important steps known as: (i) Decomposition and (ii) Reconstruction. The alcoholic or non-alcoholic EEG signal $\left(Y_{n}\right)$ is usually corrupted with other artifacts. To remove these unwanted sources from the EEG signal, we perform decomposition at the first stage and the reconstruction of the noise-free EEG signal $\left(X_{n}\right)$ is performed at the second stage.

1) Decomposition: The decomposition stage of SSA is further divided into two sub-steps named embedding and Singular Value Decomposition (SVD). Let us consider an EEG data matrix $\mathbf{G}$ of size $(K \times M)$ as mentioned in (1), where $(K=N-L+1), \mathrm{N}$ represents number of samples and $\mathrm{L}$ represents the length of the window [13].

$$
\boldsymbol{G}=\left(\begin{array}{cccc}
s_{1} & s_{2} & \ldots & s_{K} \\
s_{2} & s_{3} & \ldots & s_{K+1} \\
\vdots & \vdots & \vdots & \vdots \\
s_{L} & s_{L+1} & \cdots & s_{N}
\end{array}\right)
$$

Secondly, SVD is performed on the matrix G. The implementation of SVD on $\mathbf{G}$ can be represented as:

$$
\mathbf{G}=\mathbf{V D U}^{T}
$$

where, $\mathbf{V}$ and $\mathbf{U}$ are the unitary matrices and $\mathbf{D}$ is a diagonal matrix.

For the most part, SVD is performed to locate the symmetrical eigen vectors. The covariance matrix $\mathbf{C}$ for information matrix $\mathbf{G}$ is given by $\mathbf{C}=\mathbf{G G}^{T}$, which speak to the relating eigenvalues and eigenvectors as $\lambda_{1}, \lambda_{2}, \ldots, \lambda_{M}$ and $\mathbf{V}_{1}, \mathbf{V}_{2}, \ldots, \mathbf{V}_{M}$ individually. The $i_{\text {th }}$ component of $\mathbf{U}$ can be expressed as in (3),

$$
\mathbf{u}_{i}=\frac{\mathbf{G}^{T} \mathbf{v}_{i}}{\sqrt{\lambda_{i}}}
$$

where, $i=1,2, . ., M$. Then, the trajectory matrix can be expressed as,

$$
\mathbf{G}=\sum_{i=1}^{M} \mathbf{G}_{i}=\sum_{i=1}^{M} \sqrt{\lambda_{i}} \mathbf{v}_{i} \mathbf{u}_{i}^{T}
$$




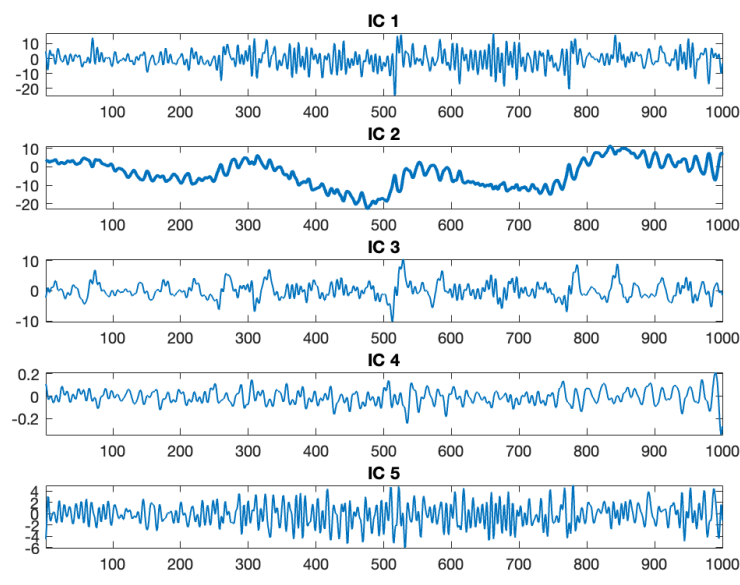

Fig. 3: ICs of normal EEG signal

where trajectory matrix $\mathbf{G}_{i}$ can be defined as

$$
\mathbf{G}=\mathbf{v}_{i} \mathbf{v}_{i}^{T} \mathbf{G}
$$

The term $\mathbf{v}_{i} \mathbf{v}_{i}^{T}$ in (3) forms a subspace, which is formed by the eigenvector $\mathbf{v}_{i}$.

2) Reconstruction: Constructing the SSA again comprises of two steps, which are mainly grouping and diagonal averaging. It has been observed that to construct again each elementary component for any value of L, the usual SSA fails. So in this paper we apply clustering after rebuilding each elementary component, and for reconstruction we have used the Hankelization process [14].

$$
\mathbf{G}_{i}=[g(k, p)]=s_{i}(n)
$$

where, $k$ and $p$ are the indices of the elements of $\mathbf{G}_{i}$ and $s_{i}(n)$ is the $i_{t h}$ component of $\mathbf{G}_{i}$.

To initialize this algorithm, we allocate each reconstructed elementary component to a unique class. Then to build a novel class the algorithm looks for the closest two classes and puts them together. And we keep on doing this process until the desired number of classes or the maximum dissimilitude between two classes is achieved. For comparing the classes $C_{i}$ and $C_{j}$ which have a lot of time series data, the minimum distance between the two distinct time series of each class gives the dissimilarity.

$$
D\left(C_{i}, C_{j}\right)=\min _{x \in C_{i}, y \in C_{j}} d(x, y)
$$

the distance function $\mathrm{d}$ is: $\mathbb{R}^{N} \times \mathbb{R}^{N} \rightarrow[0,1]$

$$
d(x, y)=1-\frac{|<x, y>|}{\|x|||| y\|}
$$

where, $\langle x, y\rangle=x^{T} y$ and $\|x\|=\sqrt{<x}, x>$

The autoSSA function is used to perform the hierarchical clustering on the $s_{i}(n)$ components which are, $\left[s_{1}(n), s_{2}(n), s_{3}(n), \ldots, s_{R}(n)\right]$. Finally, the $j_{t h}$ cluster components are assigned as indices of $J_{j}$ where, $j=1,2,3 \ldots, r$. In
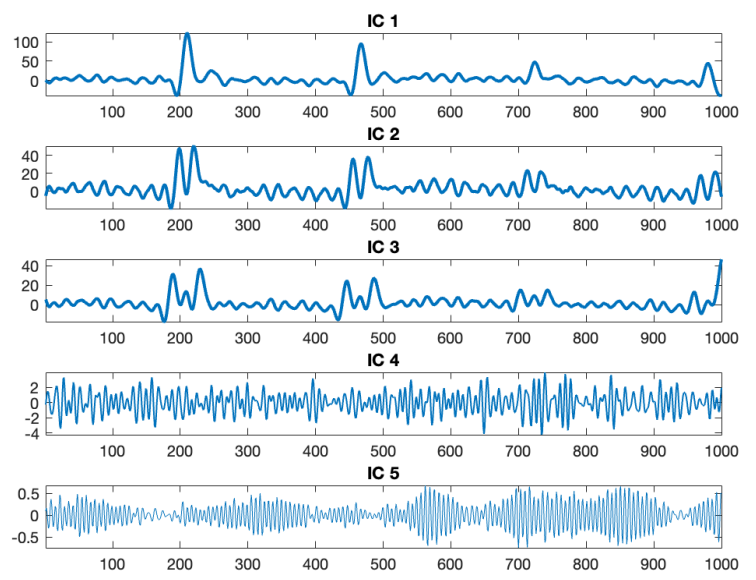

Fig. 4: ICs of alcoholic EEG signal

the last step, the components within each cluster are designated as the $j_{t h}$ reconstructed components [27].

\section{Independent component analysis (ICA)}

For blind source separation (BSS), ICA is used as it is a higher statistical algorithm. Through linear amalgamation of underlying latent variables, a set of arbitrary variables are expressed by BSS. An arbitrary vector can be denoted using $\mathbf{x}$ whose elements are a blend of $x_{1}, \ldots, x_{n}$, and similarly by $\mathbf{s}$ where the arbitrary vector is composed of elements $s_{1}, \ldots, s_{n}$. A matrix, let it be donated by $\mathbf{A}$ has elements $a_{i j}$. So by using this vector-matrix notation, we can write the above model as

$$
x=A s
$$

ICA is the name for the statistical model in Eq. (7). ICA describes that how by the process of mixing the components $s_{i}$, the observed data is created, and hence is called a generative model. Independent components cannot be directly noticed as they are latent variables. And it has been assumed that the mixing matrix is not known. So, all we observe is the arbitrary vector $\mathbf{x}$, and using $\mathbf{x}$ we must estimate both $\mathbf{A}$ and $\mathbf{s}$. This should be taken as general assumption as possible [15]. The ICs of alcoholic and non-alcoholic EEG signals are shown in Fig. 3 and Fig. 4.

\section{EXPERIMENTAL RESULTS}

Initially, we took 10000 samples of alcoholic and normal EEG data and divided it into 50 channels by setting the window length $(\mathrm{L}=70)$. After decomposition, we have applied fastICA algorithm to extract the various independent components of alcoholic and non-alcoholic EEG signals. The output of five ICs for both alcoholic and non-alcoholic EEG signals are shown in Fig. 1 and Fig. 2. The proposed method is performed on Apple MacBook powered with M1 chip. We used autoSSA and fastICA functions on MATLAB 2020b to implement sliding SSA-ICA algorithm. 
TABLE I: Computational time for sliding SSA-ICA of various classifiers (in seconds)

\begin{tabular}{c|c|c|c|c|c|c|c|c|c|c}
\hline & $\mathbf{5}$ & $\mathbf{1 0}$ & $\mathbf{1 5}$ & $\mathbf{2 0}$ & $\mathbf{2 5}$ & $\mathbf{3 0}$ & $\mathbf{3 5}$ & $\mathbf{4 0}$ & $\mathbf{4 5}$ & $\mathbf{5 0}$ \\
\hline \hline SVM & 39.30 & 40 & 41.26 & 38.35 & 24.00 & 22.62 & 27.29 & 38.38 & 25.79 & 31.08 \\
\hline KNN & 5.50 & 21.40 & 37.97 & 25.67 & 16.75 & 18.71 & 18.1 & 21.33 & 20.12 & 18.47 \\
\hline Gradient Boosting & 2.60 & 4.80 & 6.80 & 9.01 & 5.27 & 7.02 & 10.69 & 11.70 & 10.85 & 13.77 \\
\hline Ada Boost & 3.60 & 5.80 & 8.60 & 10.71 & 6.35 & 8.42 & 11.85 & 12.48 & 13.73 & 17.05 \\
\hline XGBoost & 0.68 & 3.78 & 4.14 & 5.57 & 4.29 & 6.60 & 7.46 & 8.77 & 8.50 & 9.65 \\
\hline ANN & 77.43 & 79.37 & 79.68 & 77.79 & 47.63 & 57.37 & 62.19 & 57.76 & 49.86 & 53.22 \\
\hline
\end{tabular}

TABLE II: Computational time for sliding SSA-PCA of various classifiers (in seconds)

\begin{tabular}{c|c|c|c|c|c|c|c|c|c|c}
\hline & $\mathbf{5}$ & $\mathbf{1 0}$ & $\mathbf{1 5}$ & $\mathbf{2 0}$ & $\mathbf{2 5}$ & $\mathbf{3 0}$ & $\mathbf{3 5}$ & $\mathbf{4 0}$ & $\mathbf{4 5}$ & $\mathbf{5 0}$ \\
\hline \hline SVM & 25.21 & 22.23 & 24.17 & 38.35 & 15.92 & 22.62 & 20.08 & 22.10 & 24.6 & 26.83 \\
\hline KNN & 4.18 & 13.11 & 36.16 & 22.05 & 24.16 & 24.78 & 24.74 & 25.19 & 25.18 & 22.47 \\
\hline Gradient Boosting & 2.65 & 4.87 & 6.84 & 8.9 & 11.05 & 11.06 & 14.25 & 16.08 & 18.76 & 19.42 \\
\hline Ada Boost & 3.63 & 6.13 & 8.91 & 10.75 & 6.35 & 16.33 & 19.31 & 19.48 & 21.6 & 24.33 \\
\hline XGBoost & 0.8 & 3.77 & 3.9 & 5.57 & 6.58 & 6.15 & 7.4 & 7.26 & 7.73 & 9.23 \\
\hline ANN & 85.45 & 77.68 & 79.08 & 77.79 & 76.98 & 77.03 & 78.14 & 80.48 & 78.21 & 81.22 \\
\hline
\end{tabular}

TABLE III: Comparison of different classification parameters for Sliding SSA-ICA using various machine learning models

\begin{tabular}{|c|c|c|c|c|c|c|c|c|c|c|}
\hline Independent components (ICs) & 5 & 10 & 15 & 20 & 25 & 30 & 35 & 40 & 45 & $\mathbf{5 0}$ \\
\hline \multicolumn{11}{|l|}{ Accuracy } \\
\hline KNN & 76.17 & 85.67 & 89.30 & 91.85 & 93.70 & 95.47 & 96.02 & 96.72 & 97.82 & 98.22 \\
\hline Gradient Boosting & 74.95 & 78.30 & 79.47 & 82.67 & 85.50 & 86.85 & 88.12 & 88.22 & 91.10 & 90.30 \\
\hline Ada Boost & 75.10 & 78.62 & 79.50 & 82.22 & 85.05 & 85.97 & 88.00 & 88.20 & 90.27 & 89.50 \\
\hline SVM & 67.70 & 68.51 & 71.80 & 74.28 & 77.26 & 82.55 & 84.82 & 85.02 & 88.77 & 89.10 \\
\hline XGBoost & 77.82 & 88.55 & 92.15 & 94.95 & 96.77 & 98.25 & 98.45 & 98.70 & 98.85 & 98.97 \\
\hline ANN & 76.53 & 85.72 & 91.33 & 94.05 & 95.13 & 96.50 & 97.72 & 97.43 & 97.87 & 97.37 \\
\hline \multicolumn{11}{|l|}{ Precision } \\
\hline KNN & 0.77 & 0.86 & 0.9 & 0.92 & 0.93 & 0.94 & 0.96 & 0.96 & 0.97 & 0.97 \\
\hline Gradient Boosting & 0.77 & 0.86 & 0.9 & 0.92 & 0.93 & 0.94 & 0.96 & 0.96 & 0.97 & 0.97 \\
\hline Ada Boost & 0.77 & 0.86 & 0.9 & 0.92 & 0.93 & 0.94 & 0.96 & 0.96 & 0.97 & 0.97 \\
\hline SVM & 0.72 & 0.76 & 0.78 & 0.79 & 0.82 & 0.84 & 0.85 & 0.86 & 0.89 & 0.89 \\
\hline XGBoost & 0.72 & 0.76 & 0.78 & 0.79 & 0.82 & 0.84 & 0.85 & 0.86 & 0.89 & 0.89 \\
\hline ANN & 0.79 & 0.87 & 0.91 & 0.94 & 0.95 & 0.97 & 0.98 & 0.98 & 0.98 & 0.98 \\
\hline \multicolumn{11}{|l|}{ Recall } \\
\hline KNN & 0.77 & 0.85 & 0.89 & 0.91 & 0.92 & 0.94 & 0.96 & 0.96 & 0.97 & 0.97 \\
\hline Gradient Boosting & 0.77 & 0.85 & 0.89 & 0.91 & 0.92 & 0.94 & 0.96 & 0.96 & 0.97 & 0.97 \\
\hline Ada Boost & 0.77 & 0.85 & 0.89 & 0.91 & 0.92 & 0.94 & 0.96 & 0.96 & 0.97 & 0.97 \\
\hline SVM & 0.68 & 0.71 & 0.73 & 0.76 & 0.79 & 0.83 & 0.84 & 0.85 & 0.89 & 0.89 \\
\hline XGBoost & 0.68 & 0.71 & 0.73 & 0.76 & 0.79 & 0.83 & 0.84 & 0.85 & 0.89 & 0.89 \\
\hline ANN & 0.79 & 0.87 & 0.91 & 0.94 & 0.95 & 0.97 & 0.98 & 0.98 & 0.98 & 0.98 \\
\hline \multicolumn{11}{|l|}{ F1 Score } \\
\hline KNN & 0.77 & 0.85 & 0.89 & 0.91 & 0.92 & 0.94 & 0.96 & 0.96 & 0.97 & 0.97 \\
\hline Gradient Boosting & 0.77 & 0.85 & 0.89 & 0.91 & 0.92 & 0.94 & 0.96 & 0.96 & 0.97 & 0.97 \\
\hline Ada Boost & 0.77 & 0.85 & 0.89 & 0.91 & 0.92 & 0.94 & 0.96 & 0.96 & 0.97 & 0.97 \\
\hline SVM & 0.67 & 0.69 & 0.72 & 0.75 & 0.78 & 0.82 & 0.84 & 0.85 & 0.89 & 0.89 \\
\hline XGBoost & 0.68 & 0.71 & 0.73 & 0.76 & 0.79 & 0.83 & 0.84 & 0.85 & 0.89 & 0.89 \\
\hline ANN & 0.79 & 0.87 & 0.91 & 0.94 & 0.95 & 0.97 & 0.98 & 0.98 & 0.98 & 0.98 \\
\hline
\end{tabular}

TABLE IV: Comparison of proposed algorithm with state-of-the-art works implemented on UCI database

\begin{tabular}{c|c|c}
\hline \hline Author & Models & Accuracy (\%) \\
\hline \hline Ekaputri et al. [16] & SVM & 77.80 \\
\hline Ehlers et al. [8] & Correlation dimension (CD) based discriminant analysis (DAC) & 88.00 \\
\hline Rieg et al. [17] & Random Forest & 96.67 \\
\hline Sharma et al. [18] & LS-SVM & 97.08 \\
\hline Malar et al. [19] & ELM & 87.60 \\
\hline P. Dewi Purnamasari et al. [20] & BPNN & 90.00 \\
\hline Faust et al. [28] & HOS based fuzzy sugeno classifier & 92.00 \\
\hline Kannathal et al. [29] & CD, Lyapunov exponent, entropy with DAC & 90.00 \\
\hline Acharya et al. [9] & Approximate entropy, SampleEn, Lyapunov exponent, Higher Order Spectra (HOS) with SVM & 91.70 \\
\hline L. Farsi et al. [30] & PCA + ANN & 71.00 \\
& LSTM 1 & 91.00 \\
& LSTM 2 & 93.00 \\
\hline Proposed Work & ANN & $\mathbf{9 7 . 3 7}$ \\
& KNN & $\mathbf{9 8 . 2 2}$ \\
\hline
\end{tabular}




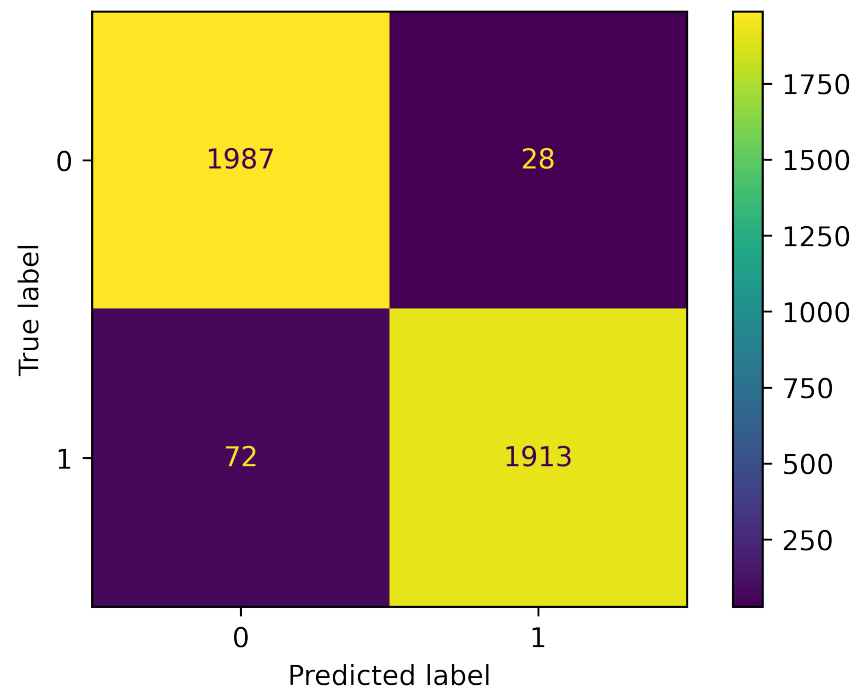

Fig. 5: Confusion matrix for KNN classifiers

We tested different machine learning models and a deep learning model to classify the EEG signals as alcoholic or nonalcoholic. The models used in for classification are AdaBoost, SVM, KNN, Gradient Boosting, XGBoost and ANN. The steps taken for implementing the models is as follows: -

- The dataset was annotated and then it was shuffled so that each data point contributes independently and there is no biasing.

- The feature selection was done in steps of 5 .

- Robust Scaler was used for the pre-processing of the attributes.

- The dataset was divided into training and testing data. The training data consisted of 16000 rows and the testing data consisted of 4000 rows.

- The above mentioned models were then trained and their performance metric were calculated using the testing data.

For gradient boosting, we took number of estimators as 100 , learning rate as 1.0 , max depth as 1 and random state as 0 . For AdaBoost classifier we have taken number of estimators as 100 and random state as 0 . We have taken rbf kernel in SVM. For XGBosst the number of estimators taken are 100. Lastly, for ANN we have used total of 8 layers. For all the hidden layers activation function used is relu and for the output layer the activation function used is sigmoid. Further the optimizer used is adam, sparse categorical crossentropy is used for calculating the loss and sparse categorical accuracy is used for calculating the accuracy. The number of epochs and batch size is 50 . Finally, we have calculated the computational time of all the classifiers for sliding SSA-ICA algorithm which is presented in Table I. To compare the computational time of proposed algorithm, we have applied PCA algorithm and the results are shown in Table II.

The classification was done using Python 3.8.5 on a PC loaded with $8 \mathrm{~GB}$ of RAM and powered with i5 processor. The accuracy of all the algorithms is shown in Fig. 7. All the performance metrics such as accuracy, precision, recall and F1 score are shown in Table III. To give a clear picture

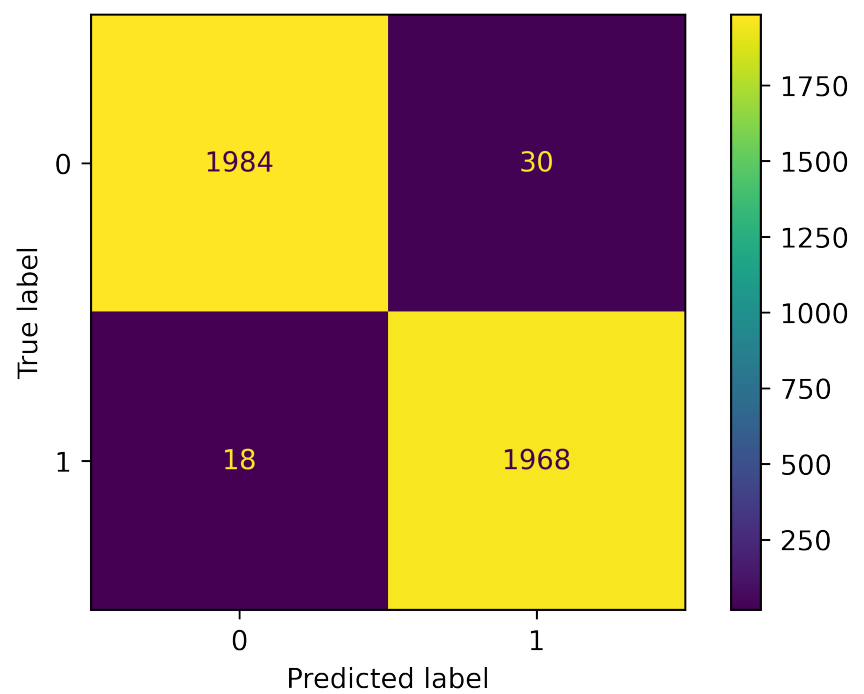

Fig. 6: Confusion matrix for XGBoost classifier

of classification between alcoholic and non-alcoholic EEG signals using sliding SSA-ICA we have plotted the confusion matrix for the best classifiers as shown in Fig. 5 and Fig. 6.

\section{DISCUSSION}

Ekatpuri et al. [16] used MWPE method for the classification of the signals, the highest accuracy was produced by the classifier Db8 with quadratic SVM. Rieg et al. [17] concluded ML models can give better accuracy if classical frequency are divided into small frequency bands. They have proved that due to this division there is a significant information content increase. In [19] Malar et al. have stated that electroencephalograph can be used to differentiate between the alcoholic and non-alcoholic signals, also it has been claimed that alcohol intake influences EEG through power spectral density analysis. For statistical feature extraction they have used wavelet transformation. Further to classify they have used Bayes, Naïve Bayes, RBNF, MLP and ELM and ELM gave the

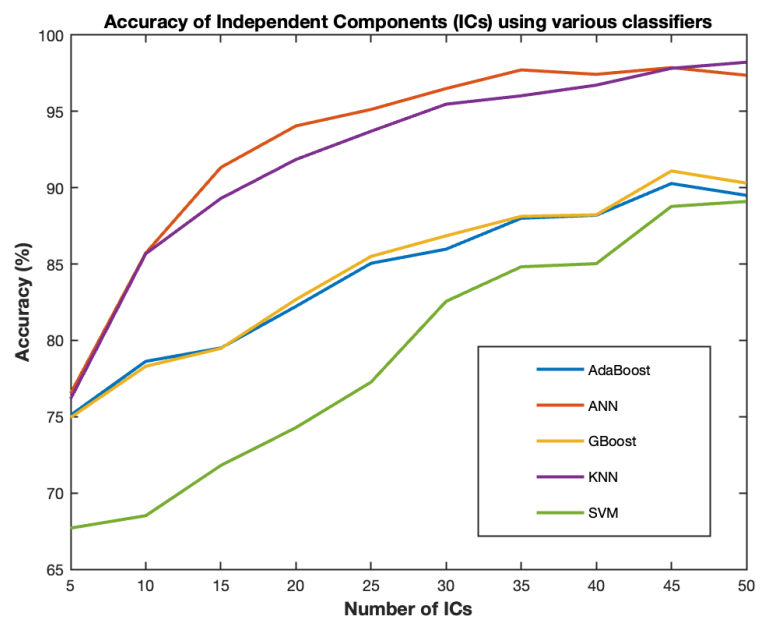

Fig. 7: Accuracy plot for various ML models 
best accuracy. Sharma et al. [18] for diagnosis of alcoholism made a new computer-aided system using DBP optimized TBOWFB. For classifying the signals, they have used LSSVM with RBF kernel and quadratic kernels. To reduce the effect of over-fitting and redundancy they have used 10-fold cross validation. The use of $\mathrm{CV}$ also ensures that the system is robust enough to be used in practical setup. Authors have used features which were extracted from DPB optimal three band FB. Also using TBOWFB they have obtained low energy of SB's. P. Dewi Purnamasari et al. [20] used relative wavelet bispectrum for feature extraction and they used 2-fold cross validation to get $90 \%$ accuracy on classification. They have stated that higher is the recognition achieved if bigger is the lag setting in the correlation calculation.

To overcome the issues discussed in the above works, we have proposed a novel algorithm to classify alcoholic and non-alcoholic EEG signals by using sliding SSA and ICA. The classification parameters show that the proposed work outperforms the recent works by very huge margins.

\section{CONCLUSION}

In this paper, we combined two algorithms named sliding SSA and ICA to identify the alcoholic features from the EEG signals. Initially, the EEG signals were decomposed using sliding SSA technique. Then, the independent components of decomposed EEG signal were calculated using ICA. Finally, the alcoholic and non-alcoholic components were given as an input to various machine learning classifiers. The classification metrics of all the models is shown in Table III. In this work, we have compared the computational complexity of machine learning classifiers using sliding SSA-ICA with PCA as shown in Table I and Table II. The proposed algorithm achieved an highest accuracy of $98.97 \%$ on XGBoost classifier. To validate the proposed algorithm, we have compared our work with the latest state-of-the-art works as shown in Table IV. In future, we are planning to detect alcoholism in EEG signals using deep learning algorithms based on statistical features.

\section{REFERENCES}

[1] D. P. Subha, P. K. Joseph, R. Acharya and C. M. Lim, "EEG signal analysis: a survey," Journal of Medical Systems. vol. 34, no.2, pp. 195212, 2010.

[2] Medical News Today. Accessed: May 29, 2018. [Online]. Available: https://www.medicalnewstoday.com/articles/157163.php

[3] R. Widadi, I. Soesanti, and O. Wahyunggoro, "EEG Classification Using Elliptic Filter and Multilayer Perceptron Based on Gamma Activity Features," in Proceedings - 2018 4th International Conference on Science and Technology, ICST 2018, 2018, vol. 1, pp. 1-5.

[4] M. Saddam, H. Tjandrasa and D. A. Navastara, "Classification of alcoholic EEG using wavelet packet decomposition, principal component analysis, and combination of genetic algorithm and neural network," 2017 11th International Conference on Information \& Communication Technology and System (ICTS), Surabaya, 2017, pp. 19-24.

[5] V. Bajaj, Y. Guo, A. Sengur, S. Siuly, and O. F. Alcin, "A hybrid method based on time-frequency images for classification of alcohol and control EEG signals," Neural Comput. Appl., vol. 28, no. 12, pp. 3717-3723, Dec. 2017.

[6] M. R. N. Kousarrizi, A. A. Ghanbari, A. Gharaviri, M. Teshnehlab, and M. Aliyari, "Classification of alcoholics and non-alcoholics via EEG using SVM and neural networks," in Proc. 3rd Int. Conf. Bioinf. Biomed. Eng., Beijing, China, Jun. 2009, pp. 1-4
[7] Y. Sun, N. Ye, and X. Xu, "EEG analysis of alcoholics and controls based on feature extraction," in Proc. 8th Int. Conf. Signal Process., Beijing, China, Nov. 2006. [Online]. Available: https://ieeexplore.ieee.org/stamp/stamp.jsp?tp=\&arnumber $=4128837$

[8] C. L. Ehlers, J. Havstad, D. Prichard, and J. Theiler, "Low doses of ethanol reduce evidence for nonlinear structure in brain activity," J. Neurosci., vol. 18, no. 18, pp. 7474-7486, Sep. 1998.

[9] U. R. Acharya, S. V. Sree, S. Chattopadhyay, and J. S. Suri, "Automated diagnosis of normal and alcoholic EEG signals," Int. J. Neural Syst., vol. 22, no. 3, Jun. 2012, Art. no. 1250011.

[10] O. Faust, W. Yu, and N. A. Kadri, "Computer-based identification of normal and alcoholic EEG signals using wavelet packets and energy measures,” J. Mech. Med. Biol., vol. 13, no. 3, Jun. 2013, Art. no. 1350033.

[11] O. Faust, U. Acharya, A. Alen, and C. Lin, "Analysis of EEG signals during epileptic and alcoholic states using AR modeling techniques," ITBM-RBM, vol. 29, no. 1, pp. 44-52, Mar. 2008.

[12] C. Blake and C. Merz, "UCI repository of machine learning databases," Dept. Inf. Comput. Sci., Univ. California, Irvine, CA, USA, Tech. Rep., 1998. [Online]. Available: https://archive. ics.uci.edu/ml/datasets/eeg+database

[13] M. Zubair, U. Kumar Naik M and R. Ahamed Shaik, "EMG Artifacts Removal from Multi-Channel EEG Signals using Multi-Channel Singular Spectrum Analysis," 2020 IEEE Applied Signal Processing Conference (ASPCON), 2020, pp. 183-187, doi: 10.1109/ASPCON49795.2020.9276684.

[14] J. Harmouche, D. Fourer, F. Auger, P. Borgnat and P. Flandrin, ’The Sliding Singular Spectrum Analysis: A Data-Driven Nonstationary Signal Decomposition Tool," in IEEE Transactions on Signal Processing, vol. 66, no. 1, pp. 251-263, 1 Jan.1, 2018, doi: 10.1109/TSP.2017.2752720.

[15] A. Hyvärinen, E. Oja, Independent component analysis: algorithms and applications, Neural Networks, Volume 13, Issues 4-5, 2000, Pages 411430, ISSN 0893-6080, https://doi.org/10.1016/S0893-6080(00)00026-5.

[16] Ekaputri, Cahyantari, Rahmat Widadi, and Achmad Rizal. "EEG Signal Classification for Alcoholic and Non-Alcoholic Person using Multilevel Wavelet Packet Entropy and Support Vector Machine.” In 2020 8th International Conference on Information and Communication Technology (ICoICT), pp. 1-4. IEEE, 2020.

[17] Rieg, Thilo, Janek Frick, Marius Hitzler, and Ricardo Buettner. "Highperformance detection of alcoholism by unfolding the amalgamated EEG spectra using the Random Forests method." In Proceedings of the 52nd Hawaii International Conference on System Sciences. 2019.

[18] Sharma, Manish, Dipankar Deb, and U. Rajendra Acharya. "A novel three-band orthogonal wavelet filter bank method for an automated identification of alcoholic EEG signals." Applied Intelligence 48, no. 5 (2018): 1368-1378.

[19] Malar, E., and M. Gauthaam. "Wavelet analysis of EEG for the identification of alcoholics using probabilistic classifiers and neural networks." International Journal of Intelligence and Sustainable Computing 1, no. 1 (2020): 3-18.

[20] P. Dewi Purnamasari, A. A. P. Ratna and B. Kusumoputro, "Relative wavelet bispectrum feature for alcoholic EEG signal classification using artificial neural network," 2017 15th International Conference on Quality in Research (QiR) : International Symposium on Electrical and Computer Engineering, 2017, pp. 154-158, doi: 10.1109/QIR.2017.8168473.

[21] L. Jiajie, K. Narasimhan, V. Elamaran, N. Arunkumar, M. Solarte and G. Ramirez-Gonzalez, "Clinical Decision Support System for Alcoholism Detection Using the Analysis of EEG Signals," in IEEE Access, vol. 6, pp. 61457-61461, 2018, doi: 10.1109/ACCESS.2018.2876135.

[22] Y. Izawa and Y. Omura, "Reliable Method to Mask Smoking Effect on Alcoholic Intake Detection Based on Photoplethysmogram Signal Analysis," in IEEE Sensors Journal, vol. 14, no. 5, pp. 1418-1424, May 2014, doi: 10.1109/JSEN.2013.2296047.

[23] Arti Anuragi, Dilip Singh Sisodia, Alcohol use disorder detection using EEG Signal features and flexible analytical wavelet transform, Biomedical Signal Processing and Control, Volume 52, 2019, Pages 384393, ISSN 1746-8094, https://doi.org/10.1016/j.bspc.2018.10.017.

[24] Shivnarayan Patidar, Ram Bilas Pachori, Abhay Upadhyay, U. Rajendra Acharya, An integrated alcoholic index using tunable-Q wavelet transform based features extracted from EEG signals for diagnosis of alcoholism, Applied Soft Computing, Volume 50, 2017, Pages 71-78, ISSN 1568-4946, https://doi.org/10.1016/j.asoc.2016.11.002.

[25] Siuly, S., Bajaj, V., Sengur, A. et al. An Advanced Analysis System for Identifying Alcoholic Brain State Through EEG Signals. Int. J. Autom. Comput. 16, 737-747 (2019). https://doi.org/10.1007/s11633-019-11787. 
[26] U. Rajendra Acharya, Vidya. S, Shreya Bhat, Hojjat Adeli, Amir Adeli, Computer-aided diagnosis of alcoholism-related EEG signals, Epilepsy \& Behavior, Volume 41, 2014, Pages 257-263, ISSN 15255050, https://doi.org/10.1016/j.yebeh.2014.10.001.

[27] Himali Singh, Rajesh Kumar Tripathy, Ram Bilas Pachori, Detection of sleep apnea from heart beat interval and ECG derived respiration signals using sliding mode singular spectrum analysis, Digital Signal Processing, Volume 104, 2020, 102796, ISSN 1051-2004, https://doi.org/10.1016/j.dsp.2020.102796.

[28] O. Faust, R. Yanti and W. Yu, "Automated detection of alcohol related changes in electroencephalograph signals", J. Med. Imag. Health Informat., vol. 3, no. 2, pp. 333-339, Jun. 2013.

[29] N. Kannathal, U. R. Acharya, C. M. Lim and P. K. Sadasivan, "Characterization of EEG-A comparative study", Comput. Methods Programs Biomed., vol. 80, no. 1, pp. 17-23, 2005.

[30] L. Farsi, S. Siuly, E. Kabir and H. Wang, "Classification of Alcoholic EEG Signals Using a Deep Learning Method," in IEEE Sensors Journal, vol. 21, no. 3, pp. 3552-3560, 1 Feb.1, 2021, doi: 10.1109/JSEN.2020.3026830.

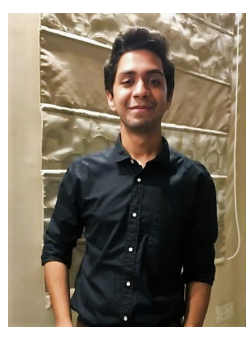

Shrey Agarwal is presently pursuing bachelor's degree in information technology at Kalinga Institute of Industrial Technology, Bhubaneswar, India. He is expecting to complete his bachelor's by May 2022. He completed a research internships at IoT Lab KIIT, Bhubaneswar, India and an industrial internship at InfoAxon, Noida, India. $\mathrm{He}$ is presently working on multiple research projects one of which is with Samsung. His research interests include Machine learning and Deep learning.

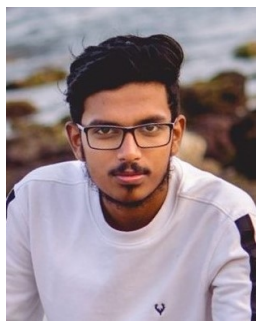

Muhammad Zubair (Student Member, IEEE) is presently pursuing bachelor's degree in electronics and communication engineering at Prasad V. Potluri Siddhartha Institute of Technology, Vijayawada, India; affiliated to JNTU Kakinada, Andhra Pradesh, India. He is expecting to complete his bachelor's by July 2021 .

$\mathrm{He}$ has published four research papers in IEEE conferences and one paper in IEEE Sensors Journal. He completed three internships at IIT Guwahati, Assam, India. He is presently an active student member at IEEE and IETE organisations. His research interests include Biomedical signal and image processing, TimeFrequency Analysis, Machine learning, Deep learning and Brain computer interface. 